\title{
Blunt Cerebrovascular Injuries: Diagnosis and Management Outcomes
}

\author{
Corie W. Wei, Walter Montanera, Daniel Selchen, Jackie Lian, \\ Christopher Stevens, Lyne Noël de Tilly
}

\begin{abstract}
Background: Blunt cerebrovascular injury (BCVI) to the carotid and vertebral arteries is a potentially devastating injury in trauma patients. The optimal management for BCVI has not been standardized. At our institution, 64-slice multi-detector computed tomographic angiography (CTA) has been used as the initial screening exam for BCVI in patients who met predefined screening criteria. The purpose of this study is to review the incidence of CTA-diagnosed BCVI in at-risk patients and to evaluate the treatment and clinical outcome of patients with BCVI. Methods: This study included trauma patients with a positive diagnosis of BCVI on CTA during a 41month study period. The medical records and relevant radiographic findings were retrospectively reviewed. Results: Twenty seven of 222 blunt trauma patients evaluated with CTA had a positive diagnosis of BCVI, with an occurrence rate of $12.2 \%$. Traumatic brain injury $(72.2 \%)$ and basal skull fractures $(55.6 \%)$ were the most frequent associated injuries with carotid trauma while $100 \%$ of blunt vertebral injuries occurred in the setting of cervical fractures. Fourteen (51.8\%) patients received medical therapy; Eleven (40.7\%) patients received conservative treatment. Endovascular treatment was attempted in a single case of vertebral arteriovenous fistula. BCVI-related stroke was found in four patients (14.8\%), one of whom developed an infarct while on medical treatment. Conclusions: BCVI is found in a significant portion of blunt trauma patients with identifiable risk factors, and screening CTA has high diagnostic yield in detecting these lesions. Medical therapy is the mainstay of treatment at our institution; however, BCVI-related stroke may occur despite treatment.
\end{abstract}

RÉSUMÉ: Diagnostic et résultats du traitement des traumatismes cérébrovasclaires contondants. Contexte : Le traumatisme cérébrovasculaire contondant (TCVC) à la carotide et aux artères vertébrales est une lésion qui peut être dévastatrice chez les patients qui ont subi un traumatisme. Le traitement optimal du TCVC n'a pas été standardisé. Dans notre institution, l'angiographie par tomodensitométrie multibarrettes a été utilisée comme examen initial de triage pour le TCVC chez les patients qui rencontraient des critères de triage prédéterminés. Objectif: Le but de cette étude était de revoir l'incidence des TCVC diagnostiqués au moyen de l'AT chez les patients à risque et d'évaluer le traitement et l'issue clinique des patients qui en sont atteints. Méthodes : Les patients qui avaient subi un traumatisme et chez qui un diagnostic de TCVC a été posé à l'AT sur une période de 41 mois ont été inclus dans cette étude. Les dossiers des patients et les constatations radiologiques pertinentes ont été révisés rétrospectivement. Résultats : Vingt-sept des $222(12,2 \%)$ patients qui avaient subi un traumatisme contondant et qui ont subi une AT, ont reçu un diagnostic de TCVC. La lésion cérébrale traumatique $(72,2 \%)$ et les fractures de la base du crâne $(55,6 \%)$ étaient les blessures les plus fréquentes associées au traumatisme de la carotide alors que $100 \%$ des blessures vertébrales contondantes sont survenues dans le contexte de fractures cervicales. Quatorze patients $(51,8 \%)$ ont été traités médicalement et 11 patients $(40,7 \%)$ ont reçu un traitement conservateur. Un traitement endovasculaire a été tenté dans un seul cas, un patient porteur d'une fistule artérioveineuse vertébrale. Quatre patients $(14,8 \%)$ ont subi un accident vasculaire cérébral en relation avec le TCVC, dont un qui a subi un infarctus alors qu'il était sous traitement médical. Conclusions : Une proportion importante des patients qui ont subi un traumatisme contondant et qui ont des facteurs de risque identifiables ont un TCVC. L'AT est un très bon moyen diagnostique pour détecter ces lésions. Le traitement médical est le traitement de base dans notre institution. Cependant, un accident vasculaire cérébral consécutif au TCVC peut survenir malgré le traitement.

Can. J. Neurol. Sci. 2010; 37: 574-579

In recent years, blunt cerebrovascular injury (BCVI) is gaining attention as an important sequela of nonpenetrating trauma to the neck and skull base. Blunt cerebrovascular injury affects approximately $1 \%$ of all blunt trauma admissions, and the reported incidence is as high as $2.7 \%$ in centres with aggressive screening protocols $\mathrm{s}^{1-8}$. The pattern of BCVI consists of vascular transection, dissection, thrombosis, and formation of pseudoaneurysm or arteriovenous fistula $(\mathrm{AVF})^{9,10}$. The majority of these injuries occur during motor vehicle accidents, with variable rates of carotid and vertebral artery involvement reported in the literature ${ }^{11-13}$. If untreated, BCVI can be fatal and result in devastating neurological sequelae, with mortality and morbidity rates reported up to $38 \%$ and $67 \%$ respectively ${ }^{14}$.

One of the difficulties in initiating treatment for BCVI is related to the variable symptomatology. While approximately
$10 \%$ of patients show focal neurological findings on initial presentation, BCVI can be asymptomatic for the first 24 hours with development of neurological deficits days to months after the initial injury ${ }^{14}$. As BCVI often occurs in the setting of multisystem trauma, BCVI-related symptoms may be masked by concurrent injuries to the head and neck. As a result, many

From the Department of Medical Imaging (CWW, WM, LNdT), Department of Neurology (DS), St. Michael's Hospital, University of Toronto, Toronto, ON;

Department of Medical Imaging (JL), McGill University, Montreal, QC; Department of Medical Imaging (CS), University of British Columbia, Vancouver, BC, Canada.

Received December 17, 2009. Final Revisions Submitted April 8, 2010. Correspondence to: Lyne Noël de Tilly, Department of Medical Imaging, St. Michael's Hospital, 30 Bond Street, Toronto, Ontario, M5B 1W8, Canada. 
authors have advocated screening protocols to identify BCVI in asymptomatic patients based on injury patterns and mechanism ${ }^{15,16}$. Although four-vessel angiogram has been considered the gold standard exam to diagnose BCVI, it is invasive, time-consuming, and not readily available ${ }^{8,17,18}$. In recent years, many centres have adopted multi-detector computed tomographic angiography (CTA) to diagnose BCVI non-invasively in the setting of trauma. Studies have shown that CTA of the neck can serve as a reliable screening modality for BCVI in patients at risk for vascular injuries ${ }^{1,3,5,19}$.

Currently, the mainstay of treatment for BCVI involves anticoagulation or antiplatelet agents ${ }^{17}$. Endovascular stent and coil embolization has been used in selective patients with pseudoaneurysm or $\mathrm{AVF}^{20}$. However, it is often difficult to prescribe treatment for BCVI on an individual basis. Medical therapy is often contraindicated in patients with extensive trauma $^{18}$ while endovascular treatment can be associated with small risks of complications ${ }^{21}$. The optimal treatment algorithm for BCVI is far from standardized.

The purpose of the current study is to evaluate the incidence of BCVI in trauma patients at risk for vascular injuries based on 64-slice multi-detector CTA. We also retrospectively examined the treatment regimen and clinical outcome in patients with a positive diagnosis of BCVI. By evaluating our institution's experience with BCVI, we hope to contribute to the development of management guidelines for this patient group.

\section{Methods}

This study was conducted at St. Michael's Hospital, a major regional trauma centre affiliated with the University of Toronto, with approval from the institutional ethics review board.

In 2006, our institution adopted 64-slice multi-detector CTA of the neck to screen for BCVI in trauma patients. Patients were selected for screening based on the Denver Criteria ${ }^{22}$ (Table 1).

All CTA scans were acquired with a 64-slice multi-detector CT (LightSpeed VCT, General Electric Healthcare, Canada). Using the Carotid CTA protocol, scans were acquired from the

\section{Table 1: Denver screening criteria for blunt cerebrovascular injury (BCVI)}

\author{
Signs/Symptoms of BCVI \\ Arterial hemorrhage \\ Cervical bruit in patient $<50$ years of age \\ Expanding cervical hematoma \\ Focal neurologic deficit \\ Neurologic exam incongruous with head CT scan findings \\ Stroke on secondary CT scan
}

Risk Factors for BCVI

High-energy transfer mechanism with:

LeFort II or III fracture

Cervical-spine fracture patterns: subluxation, fractures extending into the transverse foramen, fractures of $\mathrm{C} 1-\mathrm{C} 3$

Basal skull fracture with carotid canal involvement

Diffuse axonal injury with a Glascow Coma Scale (GCS) score $<6$

Near hanging with anoxic brain injury

$\dagger$ Blunt trauma patients were evaluated with CTA if one of the Denver criteria was satisfied; CT computed tomogram. aortic arch to the vertex after injection of Visipaque 320 (General Electric Healthcare, Canada). To reduce artifacts, all CTA scans were acquired with both arms by the flank.

We identified all patients who were evaluated with neck CTA after blunt trauma between January 1, 2006 and June 1, 2009. The medical records of patients with BCVI were retrospectively reviewed for demographics, injury mechanism, treatment, and clinical follow-up. Reports of all relevant radiographic studies were also examined to determine the grade of injury, associated injuries, development of new neurological findings, and injury progression. Blunt injury to carotid and vertebral arteries were classified according to a commonly accepted grading system ${ }^{22,23}$ (Table 2). The treatment regimen for each patient was at the discretion of the attending trauma team.

\section{RESULTS}

In the 41-month study period, 222 patients had been evaluated with neck CTA after blunt mechanisms of trauma. Twenty seven patients were found to have injury to the craniocervical vessels with an occurrence rate of $12.2 \%$. The mean age was $41.2 \pm 21.7$. Eighteen of these patients were men. The mechanisms of injury were road trauma in 19 patients (13 car occupants, 3 pedestrians, 2 ATV, and 1 motorcycle), 7 falls, and 1 assault. The average length of stay in the hospital was 23.3 days.

Eighteen carotid injuries and 12 vertebral injuries were identified. Thirteen patients had unilateral carotid involvement, and nine patients had unilateral vertebral injury. Two patients sustained concurrent carotid and vertebral injuries, and one patient had bilateral carotid injury. There was one case each of arteriovenous fistula and carotid cavernous fistula. Of the 18 carotid injuries, Grade I injury was the most common (44.4\%) while Grade IV vertebral injury was the most frequent $(41.7 \%)$. Of the carotid injuries, seven cases involved the cervical internal carotid artery (ICA), four involved the distal cervical ICA extending into the petrous portion, four involved the petrous/cavenous ICA, and one involved the common carotid artery. The grades of blunt carotid and vertebral injuries are summarized in Table 3.

Associated injuries were identified in all BCVI with the exception of one Grade II blunt carotid injury. These injuries included basal skull and calvarial fractures, cervical fractures,

Table 2: Grading of blunt cerebrovascular injury

\begin{tabular}{|c|c|}
\hline Grade & Findings \\
\hline I & $\begin{array}{l}\text { Intimal irregularity or dissection causing } \\
<25 \% \text { luminal narrowing }\end{array}$ \\
\hline II & $\begin{array}{l}\text { Dissection, raised flap, or thrombus causing } \\
>25 \% \text { luminal narrowing }\end{array}$ \\
\hline III & Pseudoaneurysm \\
\hline IV & Occlusion \\
\hline $\mathrm{V}$ & Transection \\
\hline
\end{tabular}


Table 3: Numbers of cerebrovascular injuries by grade of injury

\begin{tabular}{c|c|c}
\hline Grade & $\begin{array}{c}\text { Blunt carotid } \\
\text { injury (n= 18) }\end{array}$ & $\begin{array}{c}\text { Blunt vertebral } \\
\text { injury (n= 12) }\end{array}$ \\
\hline I & 8 & 4 \\
\hline II & 6 & 1 \\
\hline III & 2 & 1 \\
\hline IV & 1 & 5 \\
\hline V & 0 & 0 \\
\hline
\end{tabular}

facial bone fractures, and traumatic brain injury. Of the blunt carotid injuries, traumatic brain injury $(72.2 \%)$ and basal skull fractures $(55.6 \%)$ were the most frequent associated injuries. One hundred percent of blunt vertebral injures were associated with cervical fractures. In addition, all vertebral artery injuries occurred at the level of associated cervical fractures. Associated injuries in patients with BCVI are summarized in Table 4.

The treatment regimens employed in patients with BCVI included medical treatments endovascular therapy, and expectant management (Table 5). Fourteen $(51.8 \%)$ patients received medical treatment with antiplatelet and/or anticoagulation agents. Antiplatelet treatments include aspirin $81 \mathrm{mg}$ or $325 \mathrm{mg}$. Anticoagulation regimen included therapeutic doses of intravenous heparin and/or warfarin sodium. Routine prophylaxis for deep vein thrombosis (DVT) using subcutaneous fractionated heparin was given to all trauma patients without
Table 4: Associated injuries in patients diagnosed with cerebrovascular injury $(n=27)$

\begin{tabular}{l|c|c}
\hline & \multicolumn{2}{|c}{ Frequency (n) } \\
\hline & $\begin{array}{c}\text { Blunt carotid injury } \\
(\mathbf{n}=\mathbf{1 8})\end{array}$ & $\begin{array}{c}\text { Blunt vertebral } \\
\text { injury } \\
(\mathbf{n = 1 2})\end{array}$ \\
\hline Facial fracture & 3 & 1 \\
\hline Basal skull fracture & 10 & 1 \\
\hline Calvarial fracture & 5 & 12 \\
\hline Cervical fracture & 8 & 4 \\
\hline $\begin{array}{l}\text { Traumatic brain } \\
\text { injury }\end{array}$ & 13 & 0 \\
\hline None & 1 & \\
\hline
\end{tabular}

contraindications and was not considered a treatment for BCVI. Ten patients received antiplatelet treatment alone; one patient were treated with anticoagulation only; three patients received a combination of aspirin and anticoagulation. Eleven $(40.7 \%)$ patients received no treatment for vascular injuries. Seven $(25.9 \%)$ of these patients had contraindications to treatment as a result of significant hemorrhagic brain/cord injuries. In one patient with traumatic vertebral AVF, endovascular treatment was attempted involving planned trapping of the vertebral artery (VA) above and below the AVF. Treatment information could not be retrieved for one patient with Grade III carotid injury.

Of the 27 BCVI patients, four patients showed evidence of cerebral or cerebellar infarction, giving a stroke rate of $14.8 \%$. Stroke was associated with $16.7 \%$ of carotid injuries (three

Table 5: Treatment regimens for patients with BCVI

\begin{tabular}{c|c||c}
\hline & Number of Patients & Type of Injury \\
\hline Medical treatments & $14(51.8 \%)$ & \\
\hline Antiplatelet alone & 10 & \\
\hline Anticoagulation alone & 1 & \\
\hline $\begin{array}{c}\text { Both antiplatelet and } \\
\text { anticoagulation }\end{array}$ & 3 & Vertebral AVF \\
\hline & 1 & \\
\hline Endovascular treatment & 11 (40.7) & $\begin{array}{c}\text { Grade IV vertebral injury (3) } \\
\text { Grade II carotid injury (1) } \\
\text { Grade I carotid injury (3) } \\
\text { Grade I vertebral injury (3) } \\
\text { No treatment }\end{array}$ \\
\hline $\begin{array}{c}\text { Carotid cavernous fistula (1) } \\
\text { injury and contraindication to treatment) }\end{array}$
\end{tabular}

$\dagger$ Routine prophylaxis for DVT using subcutaneous fractionated heparin was given to all trauma patients without contraindications and was not considered a treatment for BCVI. 
Table 6: Treatment outcomes for patients with BCVI

\begin{tabular}{|c|c|c|c|}
\hline & $\begin{array}{l}\text { Number of } \\
\text { Patients }\end{array}$ & Clinical Outcome & Follow-up CTA \\
\hline Medical treatments & $14 \quad(51.8 \%)$ & \multirow{4}{*}{$\begin{array}{l}1 \text { patient passed away } \\
3 \text { patients developed stroke } \\
10 \text { patients showed stable or } \\
\text { improved clinical course }\end{array}$} & \multirow{4}{*}{$\begin{array}{l}\text { Follow-up CTA available in } 9 \text { patients } \\
\text { - Stable dissection in } 4 \text { patients } \\
\text { - Decreased size of pseudoaneurysm } \\
\text { in } 2 \text { patients } \\
2 \text { patients developed pseudoaneuryms } \\
\text { from ICA dissection between } 1 \text { to } 3 \\
\text { months after the initial diagnosis }\end{array}$} \\
\hline Antiplatelet alone & 10 & & \\
\hline Anticoagulation alone & 1 & & \\
\hline $\begin{array}{c}\text { Both antiplatelet and } \\
\text { anticoagulation }\end{array}$ & 3 & & \\
\hline Endovascular treatment & 1 & $\begin{array}{l}\text { aborted as a result of } \\
\text { iatrogenic dissection of the } \\
\text { contralateral vertebral artery }\end{array}$ & $\begin{array}{l}5 \text { months follow-up CTA showed } \\
\text { stable left vertebral AVF and right } \\
\text { vertebral dissection }\end{array}$ \\
\hline No treatment & $11(40.7 \%)$ & $\begin{array}{l}3 \text { patients passed away } \\
1 \text { patient presented with } \\
\quad \text { Stroke } \\
7 \text { patients showed stable or } \\
\text { improved neurological status }\end{array}$ & $\begin{array}{l}\text { Follow-up CTA in } 2 \text { patients showed } \\
\text { improvement/resolution of dissection }\end{array}$ \\
\hline
\end{tabular}

cases) and $8.3 \%$ of vertebral injuries (one case). Infarction was present in two patients on initial presentation. One patient with Grade IV injury of the right cavernous/petrous ICA presented with right middle cerebral artery (MCA) territory infarct. This patient was subsequently treated with warfarin and aspirin. One patient with Grade IV injury of the vertebral artery showed small peripheral cerebellar infarcts on the initial CT. This patient did not receive treatment for the vascular injury. Two of the four patients sustained significant hemorrhagic brain injury and developed infarcts post-admission. One patient with Grade II injury to the cervical to petrous portion of the right ICA developed multiple infarcts in the right MCA territory one day post admission. Treatment with aspirin was initiated after the development of infarct. Another patient with Grade II injury to the petrous left ICA developed infarcts in the left parietal lobe 14 days after the initial CTA. This patient received aspirin for carotid dissection prior to the development of infarct.

Four patients with BCVI (14.8\%) died during hospitalization. The average time between initial CTA diagnosis of BCVI and death was 21.5 days. All four patients sustained significant intracranial and extracranial trauma, and death could not be attributed to BCVI or BCVI-related stroke in any of the cases.

Treatment outcomes for patients with BCVI are summarized in Table 6. Seven of the 11 patients, who were not treated for BCVI, showed stable or improved neurological status during clinical follow-up and did not develop new infarcts. Three patients died during hospitalization. Follow-up CTA was only available in two of the eleven patients. One patient with Grade I ICA injury showed improvement in the degree of ICA narrowing after one month. Three-month follow-up CTA in another patient with Grade I common carotid artery (CCA) injury showed resolution of intimal flap.

Ten of the 14 patients who received medical treatment for BCVI showed stable or improved clinical course during follow- up. Follow-up CTA, ranging between 2 days and 23 months after the initial presentation, was available in nine patients. Stable dissection was found in four of the nine patients. Two of the nine patients showed decreased size of pseudoaneurysm; another two patients developed pseudoaneuryms from ICA dissection between one to three months after the initial diagnosis. None of the patients receiving medical treatment developed complications related to hemorrhage.

Attempted endovascular treatment for the patient with vertebral arteriovenous fistula was aborted as a result of iatrogenic dissection of the contralateral vertebral artery. The patient did not have neurological symptoms during an eightmonth follow-up period. A follow-up CTA after five months demonstrated stable left vertebral AVF and right vertebral dissection. The patient with carotid cavernous fistula was not considered for treatment and passed away from extensive systemic injury.

\section{Discussion}

Our results indicate that injury to vertebral and carotid arteries is a common sequela of blunt head and neck trauma, occurring in $12.2 \%$ of patients who underwent screening based on identifiable risk factors. Previous studies have shown that with the application of non-selective screening protocols, BCVI can be found in 1 to $3 \%$ of all blunt trauma patients ${ }^{14,18}$. Although screening all trauma patients for BCVI maximizes the detection rate, the implementation of such a screening protocol may be unrealistic in most centres due to the burden on the health system given its resource intensive nature. According to Level III evidence in the East Management Guidelines, screening for BCVI should be considered for asymptomatic blunt head trauma patients with identifiable risk factors ${ }^{24}$. At our institution, the selection of trauma patients for BCVI screening is based on the injury mechanism, physical signs, and associated injuries 
according to the Denver Criteria ${ }^{22,23}$. The risk-based screening mechanism has shown a high yield, with BCVI found in $27 \%$ to $44 \%$ of patients with risk factors $2,6,7,14,15$. The high incidence of BCVI in our study population shows that a risk-based selection mechanism is an effective practice to screen for BCVI in blunt trauma patients.

In terms of associated injuries, blunt carotid injuries most commonly occurred with traumatic brain injury $(72.2 \%)$ and basal skull fractures (55.6\%) while all of the blunt vertebral injuries were associated with cervical fractures. Previous studies have identified independent predictors of carotid artery injury based on multivariate regression analysis: a Glasgow Coma Score less than or equal to 6 , petrous bone fracture, diffuse axonal brain injury, and LeFort II or III fracture ${ }^{15}$. Patients with all four factors had $93 \%$ probability of having injury to the carotid artery. On the other hand, the only independent predictor of vertebral artery injury was cervical spine fracture, and patients with cervical spine fracture had $33 \%$ probability of vertebral artery injury $5,15,23$. In our study, one patient (3.7\%) with Grade II carotid injury did not have any concomitant injuries in the head and neck. The absence of associated injuries in blunt carotid trauma can be explained by the mechanistic and pathophysiologic difference between carotid and vertebral injury. Proposed mechanisms of blunt carotid artery injury include shearing forces associated with acceleration-deceleration during motor vehicle collisions, abrupt flexion that compresses the carotid artery between the spine and the mandible, and hyperextension and lateral flexion of the neck stretching the carotid artery over the lateral processes of the spine. The most common accepted mechanism for vertebral artery injury, on the other hand, is injury secondary to fractures of the transverse foramen through which the vessel courses ${ }^{25}$. As a result, isolated blunt carotid injuries without concurrent head and neck trauma are more likely. However, the absence of a cervical spine injury is not a useful predictor to rule out vascular injury ${ }^{3}$, and up to $20 \%$ of patients with BCVI may not fulfill the commonly reported screening criteria $^{8,15}$.

Most of the patients in our series $(51.8 \%)$ were managed medically with antiplatelet and/or anticoagulation agents. The most common medical regime was antiplatelet therapy. Additionally, we found a significant portion of patients $(25.9 \%)$ were not candidates for any therapy as a result of extensive concomitant hemorrhagic injury. Our data is concordant with results from a large series by Stein et al which showed a third of patients with BCVI were not candidates for therapy due to discrete contraindications ${ }^{18}$. The optimal treatment of BCVI has been a subject of debate. The use of systemic anticoagulation to reduce neurological complications of BCVI was first described by Fabian et $\mathrm{al}^{26}$ and has been adopted as standard therapy in clinical practice ${ }^{4,17,26,27}$. Previous study has shown that in even in asymptomatic, untreated patients with BCVI, the rate of neurological complications can be as high as $21.5 \%{ }^{28}$. Some authors have recommended the use of antiplatelet agents as an alternative to avoid the hemorrhagic risks associated with systemic heparin ${ }^{29,30}$. Previous studies have documented decreased stroke rate from approximately $30 \%-50 \%$ to $2 \%-10 \%$ using anticoagulation or antiplatelet therapy ${ }^{4,6,8,17,22,26,31}$. Although there have been no randomized trials, retrospective analyses suggest that both therapies are equally efficacious in reducing ischemic events ${ }^{6,8,17,30,32}$. It is often not possible to prescribe therapy for BCVI on a protocol basis, and individualized therapy is necessary depending on extent of concurrent injury. As neurological and systemic hemorrhagic injuries are common in the setting of trauma, justification of withholding treatment in patients at risk for bleeding should be addressed in future studies.

The rate of BCVI-related stroke was $14.8 \%$ in our study; stroke occurred in $16.7 \%$ of carotid injuries and $8.3 \%$ of vertebral injuries. Our results are comparable to previously published data by Stein et $a 1^{18}$. In addition, three of four patients showed evidence of infarct on presentation or within the first 24 hours of admission before the initiation of treatment. The lack of a latency period between injury and infarct has been shown in previous studies ${ }^{18,33}$. One patent with Grade II carotid injury developed infarct 14 days after admission despite medical treatment. Our data shows the development of infarct can be unpreventable in patients with BCVI. We were unable to make conclusions about treatment-related risk reductions for stroke because of the small number of patients with infarcts in our series,

Radiological follow-up was available in less than half of the patients in our study. Our data shows that blunt vascular injuries may demonstrate radiographic improvement/resolution without treatment while some lesions may progress even with medical treatment. Our results support previous conclusions that few of these lesions progress and that many will improve whether or not they are treated ${ }^{4,8,15,18}$.

The only attempted endovascular treatment in a case of vertebral arteriovenous fistula resulted in iatrogenic dissection of the contralateral vertebral artery. Endovascular techniques such as coil embolization and stent placement have gained increasing popularity in the treatment of blunt vascular injuries including pseudoaneurysm, fistula, and flow-limiting dissections. While some studies have raised concerns over the safety of endovascular techniques as a result of increased risks of stroke and stent occlusion ${ }^{17,27,34}$, other groups have reported good longterm results with endovascular treatment ${ }^{35-38}$. Specifically, endovascular occlusion has been advocated as a feasible and safe treatment for traumatic vertebral arteriovenous fistula ${ }^{39,40}$. Although our study does not negate the utility of endovascular surgery in treating selective blunt vascular lesions, medical therapy is the preferred treatment at our institution.

\section{Conclusions}

Blunt cerebrovascular injury is found in a significant portion of blunt trauma patients with identifiable risk factors, and screening 64-slice multi-detector CTA has a high diagnostic yield in detecting these lesions. Vertebral injury is more likely to be associated with other injuries, most commonly cervical fractures, while carotid injury may present as an isolated finding. While medical therapy is the preferred treatment in most cases, a significant number of patients have contraindications to treatment as a result of concomitant hemorrhagic injury. Blunt cerebrovascular injury-related stroke is a significant complication and often occurs at or shortly after presentation before treatment is instituted. Stroke may also occur despite treatment. The radiological evolution of BCVI seems unaffected by treatment. Our study is illustrative of the fact that the management of BCVI is not standardized and often depends on 
the risk tolerance of the attending physician. The high prevalence and morbidity of BCVI in trauma patients necessitates the need for a risk-based screening mechanism as well as institutional practice guidelines for the management of this patient population.

\section{REFERENCES}

1. Berne JD, Reuland KS, Villarreal DH, McGovern TM, Rowe SA, Norwood SH. Sixteen-slice multi-detector computed tomographic angiography improves the accuracy of screening for blunt cerebrovascular injury. J Trauma. 2006;60(6):1204-9; discussion 1209-10.

2. Sliker CW, Shanmuganathan K, Mirvis SE. Diagnosis of blunt cerebrovascular injuries with 16-MDCT: accuracy of wholebody MDCT compared with neck MDCT angiography. AJR Am J Roentgenol. 2008;190(3):790-9.

3. Mutze S, Rademacher G, Matthes G, Hosten N, Stengel D. Blunt cerebrovascular injury in patients with blunt multiple trauma: diagnostic accuracy of duplex Doppler US and early CT angiography. Radiology. 2005;237(3):884-92. Epub 2005 Oct 26.

4. Miller PR, Fabian TC, Bee TK, et al. Blunt cerebrovascular injuries: diagnosis and treatment. J Trauma. 2001;51(2):279-85; discussion 285-6.

5. Schneidereit NP, Simons R, Nicolaou S, et al. Utility of screening for blunt vascular neck injuries with computed tomographic angiography. J Trauma. 2006;60(1):209-15; discussion 215-6.

6. Miller PR, Fabian TC, Croce MA, et al. Prospective screening for blunt cerebrovascular injuries: analysis of diagnostic modalities and outcomes. Ann Surg. 2002;236(3):386-93; discussion 393-5.

7. Kerwin AJ, Bynoe RP, Murray J, et al. Liberalized screening for blunt carotid and vertebral artery injuries is justified. J Trauma. 2001;51(2):308-14.

8. Biffl WL, Ray CE, Jr., Moore EE, et al. Treatment-related outcomes from blunt cerebrovascular injuries: importance of routine follow-up arteriography. Ann Surg. 2002;235(5):699-706; discussion -707 .

9. Feiz-Erfan I, Horn EM, Theodore N, et al. Incidence and pattern of direct blunt neurovascular injury associated with trauma to the skull base. J Neurosurg. 2007;107(2):364-9.

10. Krings T, Geibprasert S, Lasjaunias PL. Cerebrovascular trauma. Eur Radiol. 2008;18(8):1531-45. Epub 2008 Apr 8.

11. Risgaard O, Sugrue M, D'Amours S, et al. Blunt cerebrovascular injury: an evaluation from a major trauma centre. ANZ J Surg. 2007;77(8):686-9.

12. Berne JD, Cook A, Rowe SA, Norwood SH. A multivariate logistic regression analysis of risk factors for blunt cerebrovascular injury. J Vasc Surg. 2009 Jan;51(1):57-64.

13. Goodwin RB, Beery PR 2nd, Dorbish RJ, et al. Computed tomographic angiography versus conventional angiography for the diagnosis of blunt cerebrovascular injury in trauma patients. J Trauma. 2009 Nov;67(5):1046-50.

14. Anaya C, Munera F, Bloomer CW, Danton GH, Caban K. Screening multidetector computed tomography angiography in the evaluation on blunt neck injuries: an evidence-based approach. Semin Ultrasound CT MR. 2009;30(3):205-14.

15. Biffl WL, Moore EE, Offner PJ, et al. Optimizing screening for blunt cerebrovascular injuries. Am J Surg. 1999;178(6):517-22.

16. Biffl WL. Diagnosis of blunt cerebrovascular injuries. Curr Opin Crit Care. 2003;9(6):530-4.

17. Cothren CC, Moore EE, Biffl WL, et al. Anticoagulation is the gold standard therapy for blunt carotid injuries to reduce stroke rate. Arch Surg. 2004;139(5):540-5; discussion 455-6.

18. Stein DM, Boswell S, Sliker CW, Lui FY, Scalea TM. Blunt cerebrovascular injuries: does treatment always matter? J Trauma. 2009;66(1):132-43; discussion 143-4.

19. Utter GH, Hollingworth W, Hallam DK, Jarvik JG, Jurkovich GJ. Sixteen-slice CT angiography in patients with suspected blunt carotid and vertebral artery injuries. J Am Coll Surg. 2006;203(6):838-48. Epub 2006 Oct 4.
20. Duke BJ, Ryu RK, Coldwell DM, Brega KE. Treatment of blunt injury to the carotid artery by using endovascular stents: an early experience. J Neurosurg. 1997;87(6):825-9.

21. McGuinness BJ, Moriarty M, Hope JK. Interventional radiology in the treatment of intracranial vascular injuries and fistulae. Injury. 2008;39(11):1242-8. Epub 2008 Aug 15.

22. Biffl WL, Moore EE, Ryu RK, et al. The unrecognized epidemic of blunt carotid arterial injuries: early diagnosis improves neurologic outcome. Ann Surg. 1998;228(4):462-70.

23. Biffl WL, Moore EE, Elliott JP, et al. The devastating potential of blunt vertebral arterial injuries. Ann Surg. 2000;231(5):672-81.

24. Bromberg WJ, Collier B, Diebel L, et al. Blunt Cerebrovascular Injury Practice Management Guidelines. The Eastern Association for the Surgery of Trauma; 2007; 2007.

25. Cothren CC, Moore EE, Biffl WL, et al. Cervical spine fracture patterns predictive of blunt vertebral artery injury. J Trauma. 2003;55(5):811-3.

26. Fabian TC, Patton JH, Jr., Croce MA, Minard G, Kudsk KA, Pritchard FE. Blunt carotid injury. Importance of early diagnosis and anticoagulant therapy. Ann Surg. 1996;223(5):513-22; discussion 522-5.

27. Cothren CC, Moore EE, Ray CE, Jr., et al. Carotid artery stents for blunt cerebrovascular injury: risks exceed benefits. Arch Surg. 2005;140(5):480-5; discussion 485-6.

28. Cothren CC, Biffl WL, Moore EE, Kashuk JL, Johnson JL. Treatment for blunt cerebrovascular injuries: equivalence of anticoagulation and antiplatelet agents. Arch Surg. 2009;144 (7):685-90

29. Eachempati SR, Vaslef SN, Sebastian MW, Reed RL, 2nd. Blunt vascular injuries of the head and neck: is heparinization necessary? J Trauma. 1998;45(6):997-1004.

30. Wahl WL, Brandt MM, Thompson BG, Taheri PA, Greenfield LJ. Antiplatelet therapy: an alternative to heparin for blunt carotid injury. J Trauma. 2002;52(5):896-901.

31. Biffl WL, Egglin T, Benedetto B, Gibbs F, Cioffi WG. Sixteen-slice computed tomographic angiography is a reliable noninvasive screening test for clinically significant blunt cerebrovascular injuries. J Trauma. 2006;60(4):745-51; discussion 751-2.

32. Edwards NM, Fabian TC, Claridge JA, Timmons SD, Fischer PE, Croce MA. Antithrombotic therapy and endovascular stents are effective treatment for blunt carotid injuries: results from longterm followup. J Am Coll Surg. 2007;204(5):1007-13; discussion 1014-5. Epub 2007 Mar 27.

33. Mayberry JC, Brown CV, Mullins RJ, Velmahos GC. Blunt carotid artery injury: the futility of aggressive screening and diagnosis. Arch Surg. 2004;139(6):609-12; discussion 612-3.

34. Biffl WL, Moore EE, Ray C, Elliott JP. Emergent stenting of acute blunt carotid artery injuries: a cautionary note. J Trauma. 2001; 50(5):969-71.

35. Herrera DA, Vargas SA, Dublin AB. Endovascular treatment of traumatic injuries of the vertebral artery. AJNR Am J Neuroradiol. 2008;29(8):1585-9. Epub 2008 May 22.

36. Bush RL, Lin PH, Dodson TF, Dion JE, Lumsden AB. Endoluminal stent placement and coil embolization for the management of carotid artery pseudoaneurysms. J Endovasc Ther. 2001;8(1): 53-61.

37. Higashida RT, Halbach VV, Tsai FY, et al. Interventional neurovascular treatment of traumatic carotid and vertebral artery lesions: results in 234 cases. AJR Am J Roentgenol. 1989;153 (3):577-82.

38. Redekop G, Marotta T, Weill A. Treatment of traumatic aneurysms and arteriovenous fistulas of the skull base by using endovascular stents. J Neurosurg. 2001;95(3):412-9.

39. Vinchon M, Laurian C, George B, et al. Vertebral arteriovenous fistulas: a study of 49 cases and review of the literature. Cardiovasc Surg. 1994;2(3):359-69.

40. Waitzman AA, Anderson J, Willinsky RA. Endovascular management of vertebral arteriovenous fistulas: the Toronto experience. J Otolaryngol. 1996;25(5):322-8. 